Original Research Paper

\title{
The Endoribonuclease Domain of IRE1 and its Substrate HAC1 are Structurally Linked Components of the Unfolded Protein Response in Fungi
}

\author{
Luis David Maldonado-Bonilla \\ Universidad del Mar, Institute of Genetics, Puerto Escondido, Mexico
}

Article history

Received: 16-09-2020

Revised: 14-10-2020

Accepted: 14-10-2020

Email: maldonado@ zicatela.umar.mx

\begin{abstract}
The unfolded protein response sustains the folding capacity of the endoplasmic reticulum under conditions that increase the activity of the secretory pathway. In fungi, the unfolded protein response exclusively depends on the kinase/endoribonuclease IRE1, which senses the accumulation of unfolded proteins in the endoplasmic reticulum lumen and catalyzes the committed step in the unconventional splicing of HAC1 mRNA that encodes a bZIP transcription factor. The RNase domain of the IRE1 in representative fungal species was analyzed in silico. This domain shows high conservation of the residues that are required to dimerize, catalyze HAC1 mRNA cleavage and undergo regulation by flavonols. The predicted structure of the RNase domain in $N$. crassa IRE1 is reminiscent of the active domain of $S$. cerevisiae. Sequence analysis of the HAC1 gene in a select group of ascomycetes revealed features that suggested that there were conserved mechanisms of transcriptional and post-transcriptional regulation, especially the recognition and cleavage that is mediated by IRE1. These bioinformatic analyses revealed the close and conserved relationship between IRE1 and HAC1 as a mechanism that secures endoplasmic reticulum function in fungi.
\end{abstract}

Keywords: ER Stress, Intron, Endoribonuclease, bZIP Transcription Factor

\section{Introduction}

Biogenesis of secretory proteins and plasma membrane proteins is critical for organism development and adaptation. Proteins that are synthetized at the Endoplasmic Reticulum (ER) surface are sorted to the ER lumen to be folded and undergo post-translational modifications. Once the proteins are processed, they are transferred to the Golgi apparatus and they are then loaded into vesicles that move towards the plasma membrane to be fused. Cargo proteins will then be released and secreted or inserted into the plasma membrane (Anelli and Sitia, 2008). In fungi, the secretory pathway plays prominent roles in hyphal growth, degradation of polymers for nutrition and establishment of pathogenesis and symbiosis (Lo Presti et al., 2015; Steinberg, 2007). Furthermore, knowledge of this pathway in filamentous fungi can be exploited to produce secreted recombinant proteins (Nevalainen and Peterson, 2014). Unfolded proteins are accumulated at the lumen of ER when the demands of protein secretion overcomes the processing capacity of the ER, which is known as ER stress. The Unfolded
Protein Response (UPR) is a surveillance system that senses the accumulation of unfolded protein and triggers the expression of ER components to rescue the ER function and homeostasis (Hollien, 2013). In the yeast Saccharomyces cerevisiae, the UPR depends upon activity of the Inositol-requiring enzyme 1 (Ire1p), the bZIP transcription factor Homologous to ATF-CREB1 (Haclp) and the tRNA ligase 1 (Rlg1p/Trl1p) (Hetz, 2012). Ire1p is an ER-resident enzyme with kinase and endoribonuclease (RNase) activities that is located at the cytoplasmic face of the ER. A domain in the luminal region interacts with the chaperone $\mathrm{BiP}$. When unfolded proteins accumulate in the lumen, the interaction Ire1p$\mathrm{BiP}$ is disrupted and the kinase domain is autophosphorylated and activated, which enables Ire $1 p$ dimerization (Credle et al., 2005). Aggregates of Ire1p are detected during UPR, but the dimer is the functional enzyme (Aragón et al., 2009). Because of dimerization, the two RNase domains or Kinase Extension Nuclease domains (KEN) get close to each other and catalyze the endonucleolytic cleavage of the unspliced Hacl $\left(\mathrm{Hacl}^{\mathrm{u}}\right)$ mRNA in two sites that are located in loops. Each loop is 
constituent of a secondary structure that is located in the mRNA coding region and both secondary structures form the unconventional intron. After removal of the unconventional intron, Rlg1p joins the other two halves of the Hacl mRNA. This novel mRNA is known as induced Hacl (Hacl ${ }^{\mathrm{i}}$ ) (Cox and Walter, 1996). During normal ER functioning, Hacl cannot be translated because of interaction of the unconventional intron with a segment of the 5'-Untranslated Region (5'-UTR) (Rüegsegger et al., 2001). This unconvetional splicing process enables the translation of the Haclp. Haclp dimerizes and binds to the UPR Element (UPRE) in the promoters of genes encoding luminal chaperones, peptidyl-prolyl isomerases, disulfide isomerases, enzymes of phospholipid biosynthesis and other ER components (Cox et al., 1997). To sustain the execution of UPR, Haclp autoregulates its own gene expression (Ogawa and Mori, 2004). Knowledge about the IRE1 pathway in fungi is focused on yeast species (Hernández-Elvira et al., 2018) and some models such as Aspergillus spp. (Mulder et al., 2004; Richie et al., 2009) and Neurospora crassa (Montenegro-Montero et al., 2015).

The PERK and ATF6-dependent pathways also regulate the UPR. However, in fungi the UPR operates only through the IRE1 pathway. IRE1 endoribonuclease activity and the HACl mRNA sequence and structure have a close relationship and represent a critical point at which to induce the UPR.

Here, the structural in silico analysis of the IRE1 ribonuclease domain in representative fugal species from different phyla is presented and most of them are nonmodel species. The structure of the HACl gene and transcript was also analyzed in related fungi. These novel data provide structural features that might have biological relevance and offer information about regulation of the UPR in organisms where the function of the ER and the secretory pathway are pivotal for their development and adaptive responses.

\section{Materials and Methods}

\section{Gene and Protein Sequences}

S. cerevisiae Ire1p and the human homolog of ScHac1p, X-BOX BINDING PROTEIN X (XBP1) were used as queries to retrieve the corresponding sequences of IRE1 and $\mathrm{HACl}$ from the Ensembl Fungi genome browser (https://fungi.ensembl.org/index.html) using the BLASTP algorithm (Kersey et al., 2018). The ID codes for each gene and protein are enlisted in Table 1 and 2.

\section{Sequence Alignment and Identification of Functional Domains}

The RNase domain (PFAM domain PF06479) sequence of IRE1 and the bZIP domain of HAC1 proteins were aligned using the MUSCLE algorithm (Edgar, 2004). These domains were automatically identified in the
Ensembl Fungi browser because it is linked to UniProt resource database (http://www.uniport.org) (Consortium, 2017). Identification of domains was confirmed by searches in HMMER (http://hmmer.org/), which also locates transmembrane helices (TMs) using the Phobius program (Käll et al., 2004). The TMpred server was used to confirm the presence of TM (https://embnet.vitalit.ch/software/TMPRED_form.html).

\section{Protein Modelling}

The RNase domain of NcIRE1 was modelled by swiss model (Waterhouse et al., 2018) using the structure of $S c$ Ire1p at $2.4 \mathrm{~A}^{\circ}$ resolution as template (3LJ1) (Wiseman et al., 2010) because it presents the highest identity with respect to NcIRE1. The protein 3D model was visualized and edited using UCSF Chimera 1.13 (Pettersen et al., 2004).

Table 1: List of species and sequences of IRE1 used in this study. The ID corresponds to the identification code of Ensembl Fungi (https://fungi.ensembl.org/index.html)

\begin{tabular}{|c|c|}
\hline \multicolumn{2}{|l|}{ Species ID } \\
\hline \multicolumn{2}{|l|}{ Ascomycota } \\
\hline Saccharomyces cerevisiae $S 288$ c & YHR079C \\
\hline Kluyveromyces lactis NRRL Y-1140 & KLLA0_D13266g \\
\hline Schizosaccharomyces pombe 972 & SPAC167.01 \\
\hline Saitoella complicata NRRL Y-17804 & G7K_1322-t1 \\
\hline Hortaea werneckii EXF-2000 & BTJ68_11496 \\
\hline Aureobasidium namibiae CBS 147.97 & M436DRAFT_36726 \\
\hline Aspergillus niger An76 & ABL_00408 \\
\hline Fusarium oxysporum f. sp. lycopersici 4287 & FOXG__08844 \\
\hline Trichophyton rubrum CBS 202.88 & H107_01948 \\
\hline Dactylellina haptotyla CBS 200.50 & H072_2682 \\
\hline Phaeomoniella chlamydospora UCRPC4 & UCRPC4_g03305 \\
\hline Tuber borchii Tbo3840 & B9Z19DRAFT_1100132 \\
\hline Neurospora crassa OR74A & NCU02202 \\
\hline Xylona heveae TC171 & L228DRAFT_243362 \\
\hline \multicolumn{2}{|l|}{ Basidiomycota } \\
\hline Agaricus bisporus var. burnettii JB137-S8 & AGABI1DRAFT_60620 \\
\hline Laccaria amethystina LaAM-08-1 & K443DRAFT_106542 \\
\hline Piloderma croceum F 1598 & PILCRDRAFT_316 \\
\hline Fibularhizoctonia sp. CBS 109695 & FIBSPDRAFT_934984 \\
\hline Scleroderma citrinum Foug A & SCLCIDRAFT_587916 \\
\hline Paxillus rubicundulus Ve08.2h10 & PAXRUDRAFT_822623 \\
\hline Puccina coronata var. avenae & PCANC_14392 \\
\hline Melampsora larici-populina $98 A G 31$ & MELLADRAFT_46493 \\
\hline Ustilago maydis 521 & UMAG_03841 \\
\hline Anthracocystis flocculosa Pf-1 & PFL1_06479 \\
\hline \multicolumn{2}{|l|}{ Mucoromycota } \\
\hline Mucor circinelloides $1006 \mathrm{PhL}$ & HMPREF1544_01638 \\
\hline Choanephora cucurbitarum KUS-F28377 & A0J61_02401 \\
\hline \multicolumn{2}{|l|}{ Blastocladiomycota } \\
\hline Allomyces macrogynus ATCC38327 & AMA G_00580 \\
\hline Catenaria anguillulae PL171 & BCR44DRAFT_124952 \\
\hline \multicolumn{2}{|l|}{ Chytridiomycota } \\
\hline Batrachochytrium dendrobatidis JAM81 & BATDEDRAFT_20725 \\
\hline Spizellomyces punctatus DAOM BR117 & SPPG_05208 \\
\hline \multicolumn{2}{|l|}{ Cryptomycota } \\
\hline Paramicrosporidium soccamoebae KSL3 & PSACC_01985 \\
\hline Rozella allomycis CSF55 & O9G_003659 \\
\hline \multicolumn{2}{|l|}{ Zoopagomycota } \\
\hline $\begin{array}{l}\text { Basidiobolus meristosporus CBS931.73 } \\
\text { Microsporidia }\end{array}$ & K493DRAFT_410752 \\
\hline Spraguea lophii 42_110 & SLOPH_1635 \\
\hline
\end{tabular}


Table 2: List of sequences of HAC1 of ascomycetes from different orders used in this study. The ID corresponds to the identification code of Ensembl Fungi (https://fungi.ensembl.org/index.html)

\begin{tabular}{lll}
\hline Order & Species & Gene ID \\
\hline Capnodiales & Hortaea werneckii & BTJ68_13422 \\
Dothideales & Aureobasidium namibiae & M436DRAFT_53835 \\
Eurotriales & Aspergillus niger & ABL_09977 \\
Hypocreales & Fusarium oxysporum & FOXG_15625 \\
Onygenales & Trichophyton rubrum & H107_02842 \\
Orbiliales & Dactylellina haptotyla & H072_7878 \\
Phaeomoniellales & Phaeomoniella chlamydospora & UCRPC4_g04397 \\
Pezizales & Tuber borchii & B9Z19DRAFT_475277 \\
Sordariales & Neurospora crassa & NCU01856 \\
Xylonales & Xylona heveae & L228DRAFT_249044 \\
\hline
\end{tabular}

\section{Prediction of RNA Secondary Structures}

The sequences downstream of the bZIP coding region in selected $H A C l$ mRNAs were used as queries to scan for stem-loop structures in the RNAfold server that is available in the ViennaRNA Websuite (http://rna.tbi.univie.ac.at/) (Gruber et al., 2008). Models with minimum free energy are presented in this report.

\section{Results}

The species that were selected for this study are heterogeneous in their lifestyles and habitats and they encompassed the current fungal phyla (Table 1). Some selected species are saprophytic such as the ascomycetes A. niger and N. crassa, the basidiomycete A. bisporus, the mucoromycete $M$. circinelloides and the chytridiomycete $S$. punctatus. Phytopathogens such as the ascomycete $F$. oxysporum and the basidiomycetes $U$. maydis and $P$. coronata; the halotolerant asomycete $H$. werneckii; the nematode-trapping $D$. haptotyla; the endophyte $X$. heveae; and the basidiomycete mycorrhizal fungi $L$. amethystina and $P$. croceum were included. The dermatophyte $T$. rubrum and the fish-infecting microsporidal pathogen $S$. lophii were also part of the list. The diversity of the selected organisms allows a broader understanding of IRE1 structure and function.

\section{The RNase Domain of IRE1 is Conserved Among} Fungi

IRE1 is a multidomain protein with kinase and endoribonuclease activities, its architecture is presented in (Fig. 1A). The Signal Peptide (SP) and the TM allows anchoring to the ER and membrane spanning. The wide luminal region that is located at the luminal face of the ER and the kinase and RNase domains are oriented towards the cytoplasm. The luminal region harbors a luminal domain that interacts with the chaperone BiP/GRP78 in absence of ER stress to avoid IRE1 dimerization (Zhou et al., 2006). Upon UPR, dimerization of IRE1 forms the active endoribonuclease that cleaves the mRNA substrates. To determine the conservation of the RNase domain and its potential functionality in fungi, the IRE1 homolog sequences that are representative of different fungal species were retrieved from the Ensembl Fungi genome browser and aligned using the MUSCLE algorithm. The result of the alignment is presented in Fig. 1B. The RNase domain, which is oriented towards the cytoplasm, is a trilobal structure composed of eight $\alpha$-helices (Lee et al., 2008). Residues that are involved in catalysis are located at the first and fourth helices (Lee et al., 2008) and most of the residues are conserved in fungi, regardless of the phylum to which they belong (Fig. 1B). In yeast, this loop, which is formed by a leucine, basic residues and tyrosines, is required to confer specific recognition of the $H A C l$ mRNA stem-loop structures. Basic residues are mostly conserved in fungi and in the second tyrosine that correspond to Tyr1043 in S. cerevisiae. The role of Tyr1043 in the endoribonuclease activity has been experimentally confirmed (Lee et al., 2008). Tyr1041 is conserved in yeasts such as $S$. cerevisiae, K. lactis, $S$. pombe and S. complicata, but this residue is variable in other selected ascomycetes (Fig. 1B). Species of Basidiomycota, Mucoromycota and Blastocladiomycota presented an aromatic residue in this position. Conservation of the residues that are involved in the catalysis and in the loop suggests that the IRE1 homologs that are analyzed in this study may be functional endoribonucleases that operate in a similar manner as ScIre1p. Flavonols such as quercetin increase the endoribonuclease activity of ScIre1p by electrostatic interactions with residues of the first helix at the dimerization interface in the RNase domain (Wiseman et al., 2010). Residues that are involved in recognition of quercetin are conserved in fungi (Fig. 1B). Lys992 is necessary to cleave the mRNA substrate independent of the presence of flavonols (Lee et al., 2008). An aspartate in the RNAse domain is most conserved compared to lysine and in some cases, such as for S. complicata or A. bisporus, the aspartate is replaced by glutamate. 


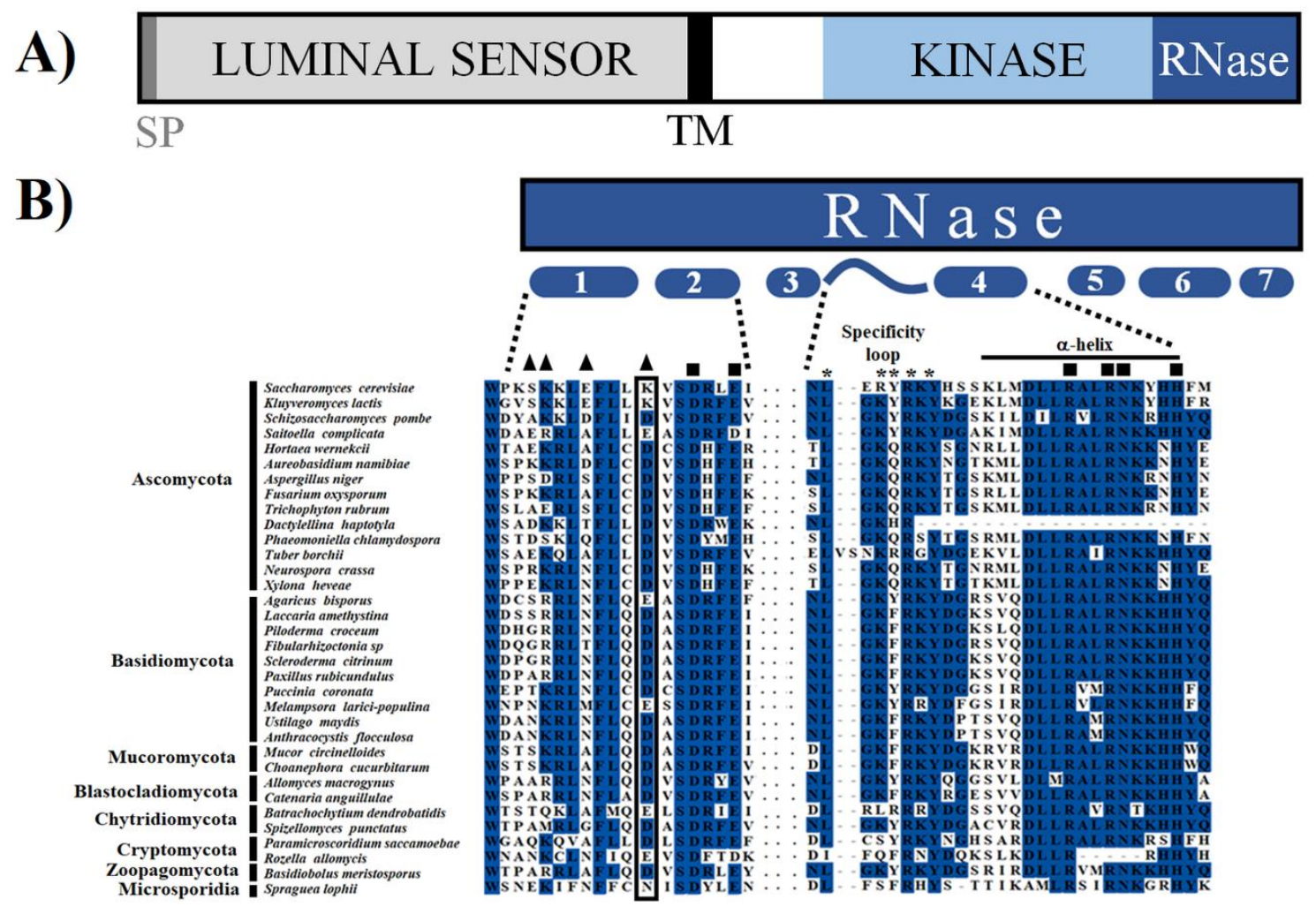

Fig. 1: Structure of the IRE1 protein. (A) Signal peptide (SP) at the N terminus precedes the luminal region of the ER and the transmembrane helix (TM), the KINASE and RNase domain are oriented towards the cytoplasm. (B) Alignment of the RNase domain of fungal species. The $8 \alpha$-helices are represented by Blue bars with white numbers. The phylum is indicated in the left of the species list. Conserved residues are shaded in blue. Black squares, asterisks and black triangles indicate residues of $S p$ Ire1p that are involved in catalysis, specificity and activation by flavonols, respectively. The K992 of SpIre1p and the other residues of this position are framed by a rectangle

\section{Predicted Structural Features of the NcIRE1 RNAse Domain}

The $N$. crassa RNase domain was modelled using the ScIre1p (3LJ1) model to define whether conservation in the RNase domain implies a similar tertiary structure (Wiseman et al., 2010). The $N$. crassa domain was selected because there has been experimental evidence of unconventional HACl splicing (Montenegro-Montero et al., 2015). Analysis of the NcIRE1 RNAse domain sequence in the secondary structure prediction servers such as Psipred or Jpred revealed that the variations in the residues do not affect the formation of $\alpha$-helices (data not shown). The predicted model as a potentially functional dimer, this model and residues that are potentially required for catalysis are shown in (Fig. 2A). In particular, the residues Asn1077 and Asp1081 constitute the first $\alpha$-helix of the domain at the interface of dimerization and they are involved in flavonol activity and regulation. The specificity loop is located at the top of the structure. Overlap of the reported and predicted ScIre1p RNase domain and predicted NcIRE1 RNase domain reveals quite similar structures (Fig. 2B), although the slight differences in residues in the first $\alpha$ helix do not compromise the structure, but they may suggest differences in the regulation of enzymatic activity among IRE1 homologs.

\section{The Structure of HACl Genes in Pezizomycotina Suggests Common Post-Transcriptional Regulation}

Unconventional splicing of HACl mRNA is reported in fungal yeast models (Hernández-Elvira et al., 2018) and filamentous models such as A. niger, A. fumigatus and N. crassa (Mulder et al., 2004; Richie et al., 2009) (Montenegro-Montero et al., 2015). Conservation of the RNase domain that is presented above suggests that IRE1 is functional and cleavage of $H A C l \mathrm{mRNA}$ at specific sites is a conserved mechanism in fungi. The $H A C l$ gene structure in species from different orders of the subphylum Pezizomycotina was analyzed to obtain insight about potential mRNA processing. To avoid a sequence bias, one species from ten different orders of Pezizomycotina were selected for this analysis. Proteins were found using the BLASTP algorithm in Ensembl Fungi, with $N c \mathrm{HAC} 1$ as a query. Identification of a HAC1 homolog often failed in other phyla because the 
best hits showed remarkably high e values or a lack of conservation in the bZIP domain. The structural HACl gene and $1000 \mathrm{bp}$ upstream of the ATG start codon are shown in Fig. 3. In A. niger, HACA/HAC1 recognizes the consensus UPR elements 5'-CAN(G/A)NTG(T/G)CCT-3' and the $10 \mathrm{mer}$ variant 5'-CACTGTCCT-3' (Mulder et al., 2006). These elements and similar sequences were identified in the potential $H A C 1$ gene promoters, suggesting a positive feedback loop (Table 3 ). In A. niger, upstream Open Reading Frames (uORF) have been identified in the 5'-UTR of HAC1/HACA mRNA and it inhibits translation of the major open reading frame. When UPR is triggered, an alternative transcription start site produces a mRNA with a shorter $5^{\prime}$-UTR that exempts the uORF, which facilitates translation (Mulder et al., 2004). Putative uORF were searched along with 5'-UTRs. Each analyzed gene presented at least one ORF. The average length of the uORFs was 56 amino acids (aa), the minimum length was a 19-aa uORF in T. rubrum HACl and the maximum length of an uORF was 137 aa, which was found in the $D$. haptotyla HACl gene (Fig. 3). None of the amino acid sequences are homologous with protein sequences or annotated as an independent ORFs in the corresponding genomes. Potential stem-loop structures were identified in the HACl mRNAs that are downstream of the region encoding for the bZIP domain (Fig. 3). A sequence encoding at least one TM was also predicted downstream of the stem-loop structures (Fig. 3).
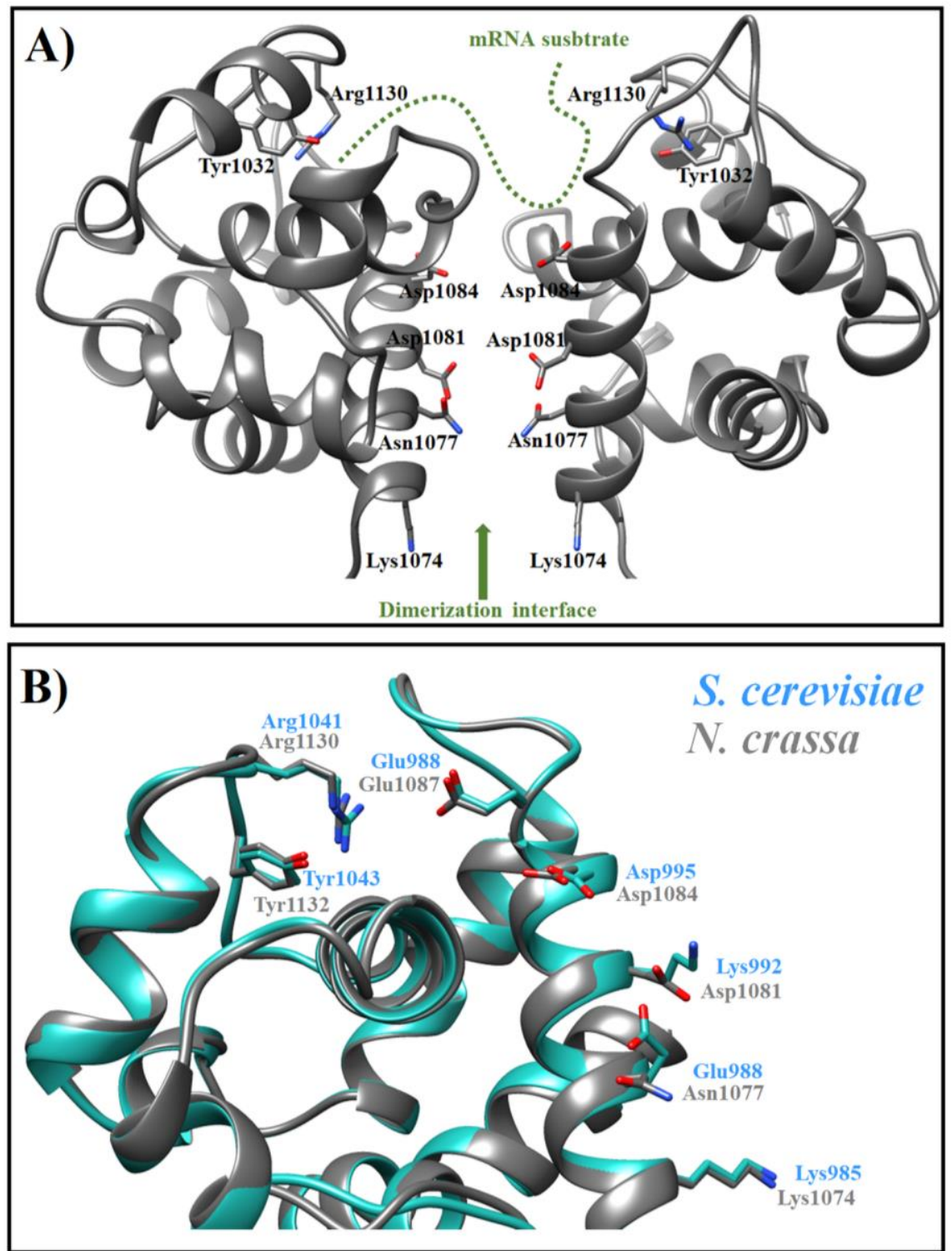

Fig. 2: Predicted tertiary structure of the dimerized RNase domain of NcIRE1. (A) The first helix required of dimerization interface and residues involved in recognition of substrate are pointed out. (B) Superimposed structures reveal conservation of the RNase domain in ScIre1p and NcIRE1 
Hortaea werneckii

Aureobasidium namibiae

Aspergillus niger

Fusarium oxysporum

Trichophyton rubrum

Dactylellina haptotyla

Phaeomoniella chlamydospora

Tuber borchii

Neurospora crassa

Xylona heveae

$\left.\Rightarrow{ }_{\text {uORF }}^{\text {Potential I UPRE }}\right|_{\text {intron }} ^{\text {CI Conventional }}$ bZIP
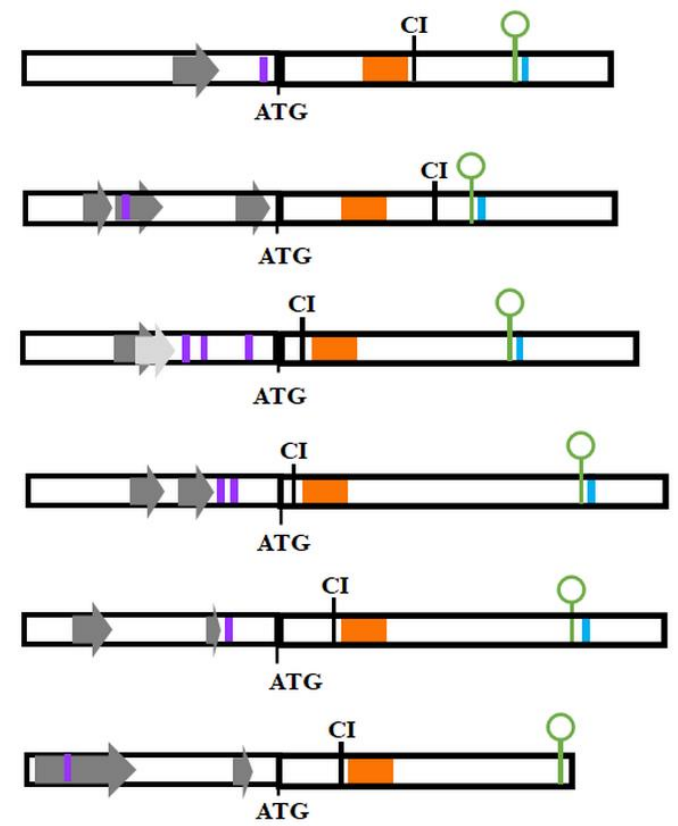

ATG
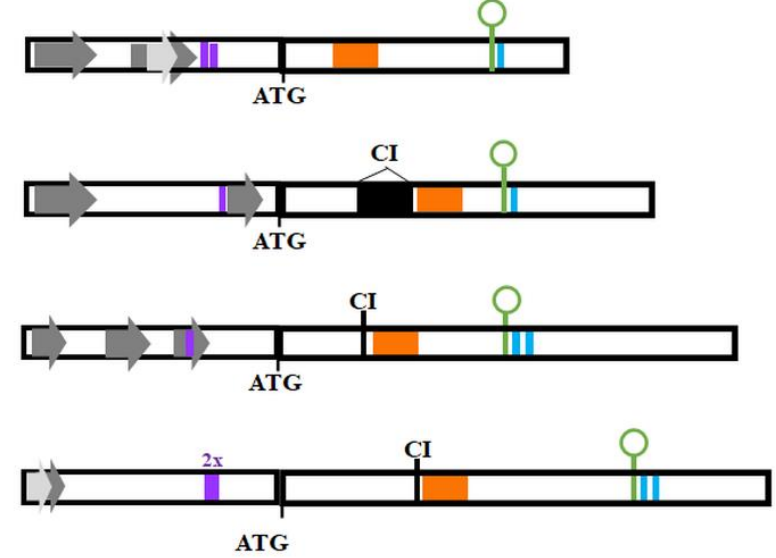

ATG

Stem-loop
Transmembrane helix

Fig. 3: Structure of the HACl gene in selected ascomycetes. Potential uORF and UPRE sequences (purple boxes) were identified in $1000 \mathrm{bp}$ upstream ATG start codon. Two-toned grey arrows were used because some of the uORF are overlapped. The coding sequence comprises the bZIP domain (orange box), conventional intron and a stem-loop sequence that is potentially recognized as an unconventional intron and a transmembrane helix. Note that the transmembrane helix coding sequence is downstream of the stem-loop

Comparison of the predicted RNA stem-loop structures reveals conservation in two motifs that are potentially recognized by IRE1 (Fig. 4). A. niger and $N$. crassa sequences were experimentally confirmed, which allows the identification of the potential IRE1 cleavage sites. The length and constitution of the sequence between the potential cleavage sites is variable (Fig. 4). The stem-loop structures are presented in Fig. 5. The structures share no similarities among them, but the nucleotides $\mathrm{G} \downarrow \mathrm{C}$ at the cleavage sites are mostly localized into loops. These RNA secondary structures might function as unconventional introns that are cleaved by IRE1 during the UPR.

\section{Structure of the HACl Proteins in Pezizomycotina}

A TM is predicted in the $\mathrm{HACl}^{\mathrm{u}}$ proteins, except in P. chlamydospora (Fig. 6A). There was no similarity in the TM sequence, but its identification suggests that, 
similar to the model plant Arabidopsis, the unspliced mRNA produces a version of the protein that is anchored to the ER membrane (Iwata and Koizumi, 2005).

The bZIP domain is a long helix that is divided by a basic motif and it interacts with DNA and a leucine zipper that mediates dimerization (Yin et al., 2017). The HAC1 protein bZIP domain in the species Pezizomycotina was aligned with the human homolog XBP1(Yoshida et al., 2001) and the human bZIP protein
FosB. The bZIP domain of FosB is crystalized and it functions as a reference to locate amino acids from a basic motif and the leucine zipper (Yin et al., 2017). The basic motif is highly conserved, but the leucine zipper is more divergent (Fig. 6B). A motif of charged amino acids, mostly basic, which were adjacent to the basic motif was highly conserved in the species Pezizomycotina in this study and it might influence the $\mathrm{HAC} 1^{\mathrm{i}}$ transcription factor activity.

Table 3: Putative AnHACA/HAC1-binding consensus sites found in 1000 bp upstream of the start codon of $H A C 1$ genes. The asterisk denotes sites similar to the consensus of $A n \mathrm{HACA} / \mathrm{HACl}$ previously reported ${ }^{27}$. The position of the sequence regarding the start codon is shown in the far right column

\begin{tabular}{lll}
\hline Species & Element & Position respect to start codon \\
\hline Hortaea werneckii & 5'-CACCTGTCCT-3' & $70-61$ \\
Aureobasidium namibiae & 5'-CAAGAGGGCCT-3'* & $569-559$ \\
Aspergillus niger & 5'-CACGTTGGCCT-3' & $359-349$ \\
& 5'-CACCTGTCCT-3' & $258-249$ \\
Fusarium oxysporum & 5'-CACCGTGGCCT-3' & $159-149$ \\
& 5'-CAACGTGTCCT-3' & $385-375$ \\
Trichophyton rubrum & 5'-CACCTGTCCT-3' & $278-268$ \\
Dactylellina haptotyla & 5'-CACGGTGGCCT-3' & $269-259$ \\
Phaeomoniella chlamydospora & 5'CAGCGTGTCCT-3'* & $756-746$ \\
& 5'-CATCCATTCCT-3'* & $247-237$ \\
Tuber borchii & 5'-CACTCTGTCCT-3'* & $231-221$ \\
Neurospora crassa & 5'-TAGTATGGCCT-3'* & $528-518$ \\
Xylona heveae & 5'-CAGCCTGGCCT-3'* & $406-396$ \\
& 5'-CAATCTGTCCT-3'* & $288-278$ \\
\hline
\end{tabular}

H. werneckii
A. namibiae
A. niger
F. oxysporum
T. rubrum
P. chlamydospora
T. borchii
N. crassa
X. heveae

\begin{tabular}{|c|c|c|}
\hline & & \\
\hline 712 & A U U C U G C A G C A A U G U U G U G C GA $\ldots$ C C U G C A G U G U & 74 \\
\hline 685 & C A U U C UG C UGCA A U G U U U G C GA $\ldots$ C C UGCAGUGU & 717 \\
\hline 634 & C A G C G G U G U U U G C GA $\ldots$ C C UGC & \\
\hline 733 & CGA U G U U G C CGA $\ldots$ C C UGC & \\
\hline 75 & C G G U G U U G U G U GA $\ldots-C C$ & 30 \\
\hline 81 & C G G U A U U G U G U A A C G G C & \\
\hline 703 & A U G U G U G A $\ldots$ & \\
\hline & & \\
\hline & & \\
\hline
\end{tabular}

Fig. 4: Alignment of the RNA sequences that are potentially recognized by the RNase domain of IRE1 of ascomycetes. Two highly conserved motifs are shaded in green. The arrow indicates the potential G $\downarrow$ C cleavage site

\section{Discussion}

The endoribonuclease activity of IRE1 depends on the RNase/KEN domain that is adjacent to the kinase domain, which is located at the $\mathrm{C}$ terminus of the protein (Fig. 1A). This RNase domain is composed of eight $\alpha-$ helices and the residues of the first and fourth helices mediate the cleavage of the RNA substrates when they dimerize (Lee et al., 2008). The loop is located between the third and fourth $\alpha$-helix in ScIre1p and it recognizes the two loop structures in Hacl mRNA; both of these loops will be subsequently cleaved at a $\mathrm{G} \downarrow \mathrm{C}$ site. RNase domain residues that are involved in catalysis and the specificity loop residues are conserved in fungi (Fig. 1B). The Lys992 in ScIre1p is crucial to cleave the Hacl mRNA and this residue is indicated by the black rectangle in Fig. 1B. Among the RNase domain sequences that were analyzed in this research, K992 is only conserved in the yeast $K$. lactis. This residue is substituted by acid amino acids in the rest of the sequences, including $S$. pombe, which lacks a HAC1 homolog (Guydosh et al., 2017). The exception is the $S$. lophii RNase domain, which has an asparagine in this position. The substitution K992D in ScIre1p decreases the endoribonuclease activity (Lee et al., 2008), suggesting that the basic nature of lysine is required for 
catalysis. Paradoxically, the acid residue is more conserved in fungi. K992 in ScIre1p also forms hydrogen bonds with E988 (Wiseman et al., 2010). This residue is highly divergent in the analyzed sequences and such divergence might be necessary to set electrostatic interactions with other residues. It will be worth comparing the $S c$ Irelp RNase domain activity against a RNase domain that harbors an acid residue in this position because it will define the function of this substitution. The mutations K985A and K992L in the ScIre1p RNase domain attenuate endoribonuclease activity and activation by flavonols such as quercetin. Quercetin is placed at the dimerization interface of the ScIre1p RNase domain and may boost its activity by clinching the dimerization of the RNase domains. Interaction with flavonols might have biological implications. Favonols are secondary metabolites that are broadly produced by plants and they have roles in the interaction between pathogens and symbionts (Buer et al., 2010). A group of RNase domains from phytopathogens such as $F$. oxysporum or mycorrhizae such as $L$. amethystina were analyzed and showed conservation in the residues in the first $\alpha$-helix that is involved in flavonol binding. The RNase domain of IRE1 in these organisms might be altered by flavonols that are produced by plants during pathogenic or beneficial interactions. This potential regulation of the endoribonuclease activity mediated by flavonols might affect the fungal protein secretion that is necessary to set the interaction with plants.

To determine whether the $\alpha$-helices are distinctive to the RNase domain of IRE1, the corresponding $N$. crassa domain was modelled (Fig. 2A). This protein was selected because there is experimental evidence of unconventional splicing of NcHACl mRNA; thus, $N$. crassa has a functional IRE1 (Montenegro-Montero et al., 2015). The predicted structure conserves the $\alpha$-helices that are involved in catalysis, dimerization and interaction with flavonols as well as and the specificity loop that recognizes the substrate. Figure 2B illustrates the overall structure of the RNase domain of $N c$ IRE1 compared to the respective domain of $S c$ IRE1p. Regardless of the differences in the residues of the first $\alpha$-helix, this secondary structure may be also formed in $N$. crassa and constitutes part of a potentially active enzyme. The NcIRE1 specificity loop harbors the residue Y1132, which is equivalent to the Y1043 of ScIre1p (Lee et al., 2008). The mutation Y1043A in the ScIre1p specificity loop dramatically decreases the endoribonuclease activity (Lee et al., 2008) and therefore, the conserved Y1132 in NcIRE1 may be critical to catalysis.

The ascomycetes $H A C l$ gene structure from different orders was analyzed in silico to determine whether their mRNAs are substrates of IRE1. The ascomycetes that were selected belonged to the Pezizomycotina subphylum and included A. niger and $N$. crassa. Schemes of the HACl genes illustrates the conservation of the sequence encoding bZIP domain (Fig. 3), a TM at the $\mathrm{C}$ terminus and a stem-loop sequence in the nearby of the TM-coding sequences. Analysis of sequences upstream of the start codon detected a UPRE consensus in A. niger and suggests hypothetical positive feedback that potentiates $H A C 1$ expression upon UPR (Ogawa and Mori, 2004). The uORFs encoded in the 5'-UTR attracts ribosomes as a mechanism to attenuate the translation of the major ORF (Hood et al., 2009). In A. niger, an uORF encoding 44 amino acids is excluded from the HACA/HACl mRNA upon ER stress by truncation of the 5'-UTR (Mulder et al., 2004). A similar condition is reported in A. fumigatus (Richie et al., 2009). Inspection of the 1000 bps that are upstream from the start codon identified a short ORF with no homology to the fungal polypeptides that might function as uORFs, but they have to be experimentally validated, especially the ORFs near the start codon.

Sequences that are reminiscent of the unconventional intron were identified in the group of predicted $\mathrm{HACl}$ mRNAs (Fig. 4). Two 6-nt motifs are highly conserved in the transcripts, except for D. haptotyla, which has stem-loop structures without the motifs. It was excluded from Fig. 4. Both motifs harbor the $\mathrm{G} \downarrow \mathrm{C}$ site that is cleaved by IRE1. The composition and sequence length between motifs are variable. The presence of the $\mathrm{G} \downarrow \mathrm{C}$ sites inside the loops is the factor that enables cleavage (Lee et al., 2008). Regulated IRE1-Dependent Decay of mRNA (RIDD) is a pathway of mRNA degradation that is conserved in eukaryotes and it prevents the protein load for maintaining the protein folding demand of the ER (Maurel et al., 2014). Substrates of RIDD are cleaved by IRE1, which recognizes a sequence that is similar to the motifs of the HACl unconventional intron. However, IRE1 activity might not be specific to loops because the human IRE1 $\alpha$ cleaves miRNA precursors at $\mathrm{G} \downarrow \mathrm{C}$ sites that are localized in stems or double-stranded RNA (dsRNA) segments (Upton et al., 2012).

Figure 5 shows the secondary structures that are predicted in the potential unconventional introns. Stemloop structures are formed in those mRNAs and the potential cleavage sites are mostly located inside loops. They are likely recognized and cleaved by IRE1 enzymes and its RNase domain conservation and functionality were previously discussed. The first $\mathrm{G} \downarrow \mathrm{C}$ site in $H$. werneckii and the two sites in $P$. chlamydospora are examples of potential cleavage sites that are situated in dsRNA (Fig. 5). The affinity of fungal IRE1 for the $\mathrm{G} \downarrow \mathrm{C}$ sites in $H A C l$ dsRNA segments is unknown, but the activity of human IRE1 $\alpha$ towards the miRNA precursors might indicate that loops harboring the $\mathrm{G} \downarrow \mathrm{C}$ sites are not the only conditional factors for $H A C 1 \mathrm{mRNA}$ cleavage. 


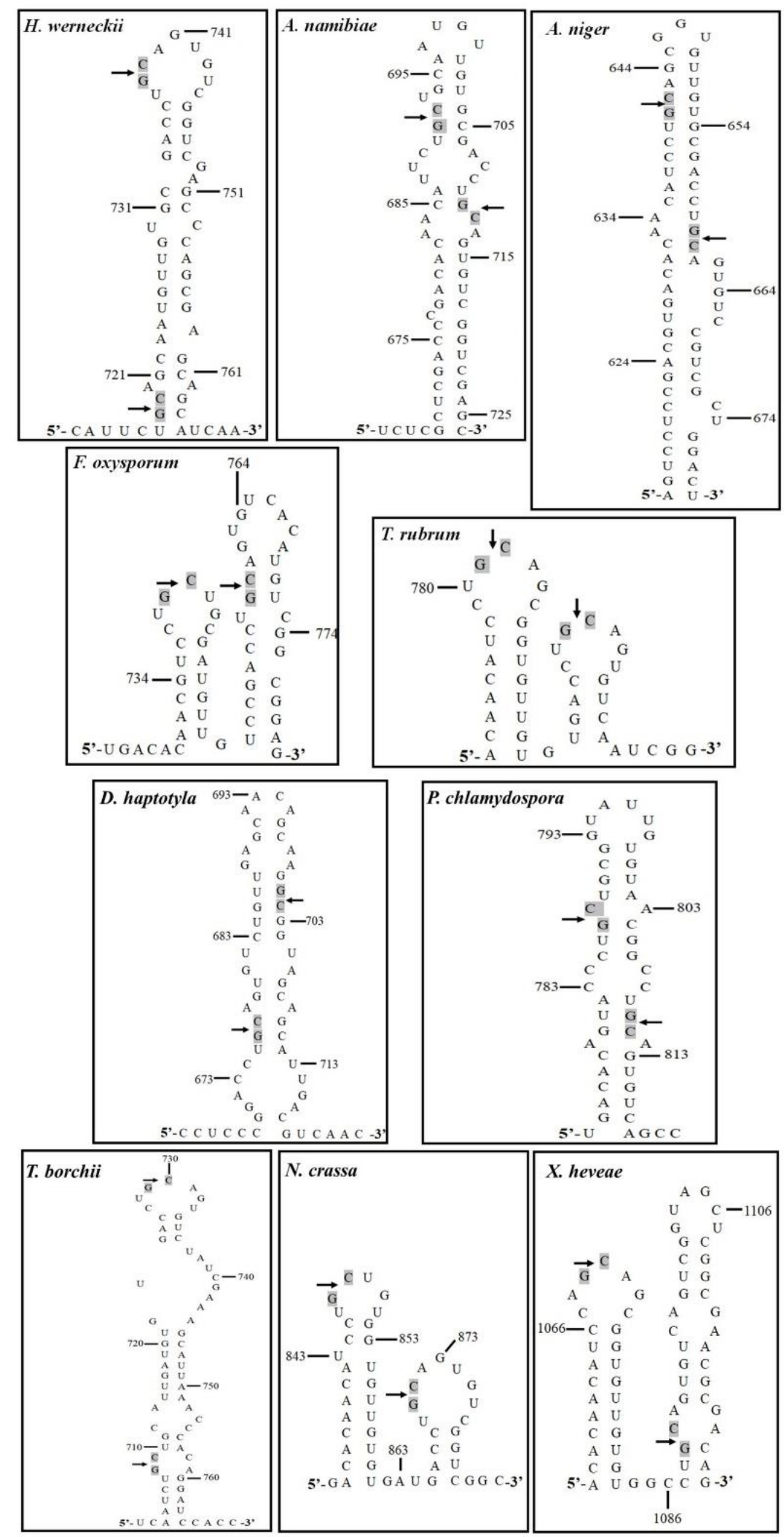

Fig. 5: $\quad$ Predicted stem-loop structures of $H A C 1$ mRNAs in ascomycetes. The arrows show the potential G $\downarrow$ C cleavage site, which are shaded in grey. Numbers indicate the position of the nucleotide in the mature mRNA 


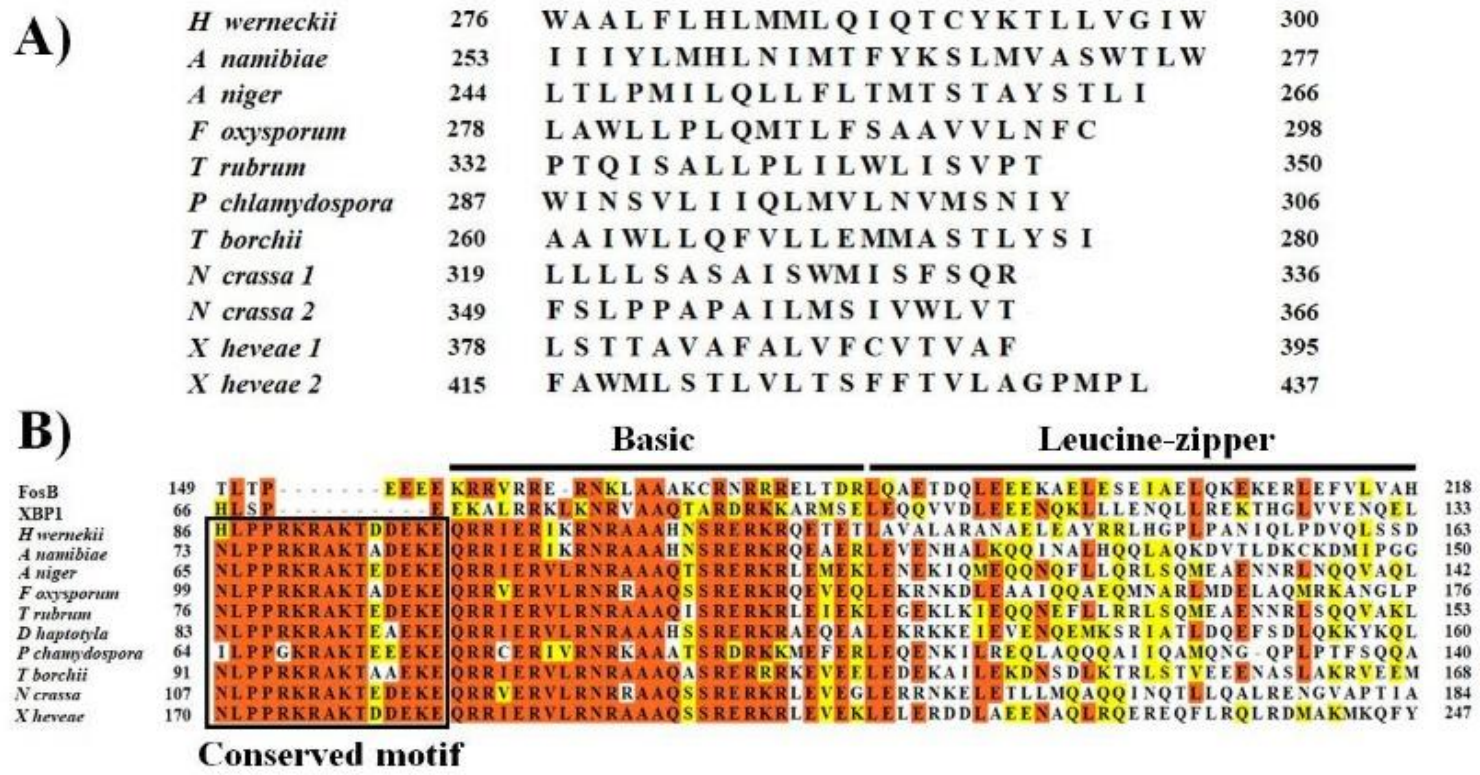

Fig. 6: Features of HAC1 proteins of ascomycetes. (A) Predicted TM with no similarity among them. Two TM in tandem were identified in $N c \mathrm{HAC1}$ and $\mathrm{XhHAC1}$. (B) Alignment of the bZIP domains of the human FOSB and Xbp1 proteins and the HAC1 of ascomycetes. Basic and leucine zipper motifs of the bZIP are marked. The black square harbors the conserved motif of ascomycetes. Identical residues are shaded in orange and similar residues are shaded in yellow. Numbers indicate the position of the residue

The putative TM that was identified in the selected group of $\mathrm{HACl}$ proteins suggests that upon ER homeostasis, inactive transcription factors accumulate in the ER membrane to avoid UPR-related gene expression, which is similar to the transcription factor ATF6 in mammals (Hollien, 2013). The ER-resident ATF6 is translocated to undergo proteolysis in the Golgi apparatus upon UPR. After lysis of its TM, ATF6 becomes active and translocates to the nucleus to activate transcription of UPR-responsive genes. A mechanism that is similar to the factors bZIP17/28 of the plant Arabidopsis is reported (Kim et al., 2018). Fungi lack an ATF6 homolog and HAC1 proteolysis has not been reported. Thus, unconventional splicing of the transcript $\mathrm{HAC}^{\mathrm{u}}$ that causes a frameshift might be the mechanism that generates the active $\mathrm{HACl}^{\mathrm{i}}$ and conservation of the components of this mechanism have been identified in the present report.

No similarities in the sequence of the putative TMs were identified in the selected group of HAC1 proteins and two TMs were detected in the HAC1 proteins of $N$. crassa and $X$. heveae (Fig. 6A). This finding might be molecularly relevant because the TMs are located at the $\mathrm{C}$ terminus and the corresponding TM-coding sequence is always downstream of the IRE1-cleavege sites. The cleavage and ligation change the open reading frame and then, the reading of the TM-coding sequence is avoided. The unrelated TMs that are found in HAC1 proteins suggest that mutations that accumulated during the course of HACl gene evolution in independent species enables the diversification of TMs, but a selection pressure drove the secondary structure and position of the IRE1-cleavege sites.

HAC1 proteins of Pezizomycotina have a unique motif that is adjacent to the bZIP domain (Fig. 6B). The $S c \mathrm{Hac}^{\mathrm{i}}$ binds to the UPRE-1 (5'-GGACAGCGTGTC$3^{\prime}$ ) and the UPRE-2 (5'TACGTG-3') and such binding is influenced by the residues that are adjacent to the basic motif of the bZIP domain (Fordyce et al., 2012). However, heterodimerization with other bZIP factors such as Gen $4 p$ also affect the in vivo recognition of targets. The homolog of HAC1 in humans, XBP1, binds to diverse sequences such as the UPRE (5'GACGTG[G]-3'), the CCACG-box and the ACTG-core (Acosta-Alvear et al., 2007). Heterodimerization, as in the case of XBP1 and the neighboring residues to the bZIP domain might impact DNA binding. Therefore, variations in the composition of UPRE in the UPRresponsive genes might coevolved with the DNA binding activity and heterodimerization of HAC1.

\section{Conclusion}

The bioinformatic analyses of selected fungal proteins and genes showed the catalytic domain of IRE1 and the potential mRNA substrate are the evolutionary conserved components of the UPR that operate similarly as $S c$ Ire1p. 
The cis-acting elements in promoters and potential uORFs identified in HAC1 genes of selected ascomycetes are hints of conserved feedback of transcriptional regulation and translational control. The conservation of the potential cleavage motifs found within secondary structures of $H A C l$ mRNAs might imply the presence of unconventional introns and the adjacent downstream sequence encoding TM suggests that the cleavage of $H A C 1 \mathrm{mRNA}$ mediated by IRE1 is a regulatory mechanism that operate to ameliorate the ER function in the UPR in fungi, in which the secretory pathway plays prominent roles in development and communication with other organisms.

\section{Acknowledgment}

This work was supported by the Program Cátedras CONACYT from the National Council of Science, Mexico (CONACYT), Grant No. 538.

\section{Ethics}

The author declares no ethical issues may arise by this publication.

\section{References}

Acosta-Alvear, D., Zhou, Y., Blais, A., Tsikitis, M., Lents, N. H., Arias, C., ... \& Dynlacht, B. D. (2007). XBP1 Controls Diverse Cell Type- and Condition-Specific Transcriptional Regulatory Networks. Molecular Cell, 27(1), 53-66.

Anelli, T., \& Sitia, R. (2008). Protein quality control in the early secretory pathway. EMBO Journal, 27(2), 315-327.

Aragón, T., van Anken, E., Pincus, D., Serafimova, I. M., Korennykh, A. V, Rubio, C. A, \& Walter, P. (2009). Messenger RNA targeting to endoplasmic reticulum stress signalling sites. Nature, 457(7230), 736-740.

Buer, C. S., Imin, N., \& Djordjevic, M. A. (2010). Flavonoids: New roles for old molecules. Journal of Integrative Plant Biology, 52(1), 98-111.

Consortium, T. U. (2017). UniProt: the universal protein knowledgebase. Nucleic Acids Research, 45(D1), D158-D169.

Cox, J S, \& Walter, P. (1996). A novel mechanism for regulating activity of a transcription factor that controls the unfolded protein response. Cell, 87(3), 391-404.

Cox, Jeffery S., Chapman, R. E., \& Walter, P. (1997). The unfolded protein response coordinates the production of endoplasmic reticulum protein and endoplasmic reticulum membrane. Molecular Biology of the Cell, 8(9), 1805-1814.
Credle, J. J., Finer-Moore, J. S., Papa, F. R., Stroud, R. M., \& Walter, P. (2005). On the mechanism of sensing unfolded protein in the endoplasmic reticulum. Proceedings of the National Academy of Sciences of the United States of America, 102(52), 18773-18784.

Edgar, R. C. (2004). MUSCLE: multiple sequence alignment with high accuracy and high throughput. Nucleic Acids Research, 32(5), 1792-1797.

Fordyce, P. M., Pincus, D., Kimmig, P., Nelson, C. S., El-Samad, H., Walter, P., \& DeRisi, J. L. (2012). Basic leucine zipper transcription factor Hac1 binds DNA in two distinct modes as revealed by microfluidic analyses. Proceedings of the National Academy of Sciences of the United States of America, 109(45).

Gruber, A. R., Lorenz, R., Bernhart, S. H., Neuböck, R., \& Hofacker, I. L. (2008). The Vienna RNA websuite. Nucleic Acids Research, 36(Web Server issue), 70-74.

Guydosh, N. R., Kimmig, P., Walter, P., \& Green, R. (2017). Regulated Ire1-dependent mRNA decay requires no-go mRNA degradation to maintain endoplasmic reticulum homeostasis in $\mathrm{S}$. pombe. ELife, 6, e29216.

Hernández-Elvira, M., Torres-Quiroz, F., EscamillaAyala, A., Domínguez-Martin, E., Escalante, R., Kawasaki, L., ... \& Coria, R. (2018). The Unfolded Protein Response Pathway in the Yeast Kluyveromyces lactis. A Comparative View among Yeast Species. Cells, 7(8), 106.

Hetz, C. (2012). The unfolded protein response: controlling cell fate decisions under ER stress and beyond. Nature Reviews Molecular Cell Biology, 13(2), 89-102.

Hollien, J. (2013). Evolution of the unfolded protein response. Biochimica et Biophysica Acta Molecular Cell Research, 1833(11), 2458-2463.

Hood, H. M., Neafsey, D. E., Galagan, J., \& Sachs, M. S. (2009). Evolutionary Roles of Upstream Open Reading Frames in Mediating Gene Regulation in Fungi. Annual Review of Microbiology, 63(1), 385-409.

Iwata, Y., \& Koizumi, N. (2005). An Arabidopsis transcription factor, AtbZIP60, regulates the endoplasmic reticulum stress response in a manner unique to plants. Proceedings of the National Academy of Sciences of the United States of America, 102(14), 5280-5285.

Käll, L., Krogh, A., \& Sonnhammer, E. L. L. (2004). A combined transmembrane topology and signal peptide prediction method. Journal of Molecular Biology, 338(5), 1027-1036. 
Kersey, P. J., Allen, J. E., Allot, A., Barba, M., Boddu, S., Bolt, B. J., ... Yates, A. (2018). Ensembl Genomes 2018: An integrated omics infrastructure for non-vertebrate species. Nucleic Acids Research, 46(D1), D802-D808.

Kim, J. S., Yamaguchi-Shinozaki, K., \& Shinozaki, K. (2018). ER-Anchored transcription factors bZIP17 and bZIP28 regulate root elongation. Plant Physiology, 176(3), 2221-2230.

Lee, K. P. K., Dey, M., Neculai, D., Cao, C., Dever, T. E., \& Sicheri, F. (2008). Structure of the Dual Enzyme Ire1 Reveals the Basis for Catalysis and Regulation in Nonconventional RNA Splicing. Cell, 132(1), 89-100.

Lo Presti, L., Lanver, D., Schweizer, G., Tanaka, S., Liang, L., Tollot, M., ... \& Kahmann, R. (2015). Fungal Effectors and Plant Susceptibility. Annual Review of Plant Biology, 66(1), 513-545.

Maurel, M., Chevet, E., Tavernier, J., \& Gerlo, S. (2014). Getting RIDD of RNA: IRE1 in cell fate regulation. Trends in Biochemical Sciences, 39(5), 245-254.

Montenegro-Montero, A., Goity, A., \& Larrondo, L. F. (2015). The bZIP transcription factor HAC-1 is involved in the unfolded protein response and is necessary for growth on cellulose in Neurospora crassa. PLoS ONE, 10(7), e0131415.

Mulder, H. J., Saloheimo, M., Penttilä, M., \& Madrid, S. M. (2004). The transcription factor HACA mediates the unfolded protein response in Aspergillus niger and up-regulates its own transcription. Molecular Genetics and Genomics, 271(2), 130-140.

Mulder, Harm J., Nikolaev, I., \& Madrid, S. M. (2006). HACA, the transcriptional activator of the unfolded protein response (UPR) in Aspergillus niger, binds to partly palindromic UPR elements of the consensus sequence 5'-CAN(G/A)NTGT/GCCT-3'. Fungal Genetics and Biology, 43(8), 560-572.

Nevalainen, H., \& Peterson, R. (2014). Making recombinant proteins in filamentous fungi- Are we expecting too much? Frontiers in Microbiology, 5(FEB), 75.

Ogawa, N., \& Mori, K. (2004). Autoregulation of the HAC1 gene is required for sustained activation of the yeast unfolded protein response. Genes to Cells, 9(2), 95-104.

Pettersen, E. F., Goddard, T. D., Huang, C. C., Couch, G. S., Greenblatt, D. M., Meng, E. C., \& Ferrin, T. E. (2004). UCSF Chimera — A Visualization System for Exploratory Research and Analysis. Journal of Computational Chemistry, 25(13), 1605-1612.
Richie, D. L., Hartl, L., Aimanianda, V., Winters, M. S., Fuller, K. K., Miley, M. D., ... \& Askew, D. S. (2009). A role for the unfolded protein response (UPR) in virulence and antifungal susceptibility in Aspergillus fumigatus. PLoS Pathogens, 5(1), e1000258.

Rüegsegger, U., Leber, J. H., \& Walter, P. (2001). Block of HAC1 mRNA translation by long-range base pairing is released by cytoplasmic splicing upon induction of the unfolded protein response. Cell, 107(1), 103-114.

Steinberg, G. (2007). Hyphal growth: A tale of motors, lipids and the spitzenkörper. Eukaryotic Cell, 6(3), $351-360$.

Upton, J.-P., Wang, L., Han, D., Wang, E. S., Huskey, N. E., Lim, F. R., ... \& Oakes, S. A. (2012). IRE1alpha cleaves select microRNAs During ER Stress to derepress translation of proapoptotic Caspase-2. Science, 338(6108), 818-822.

Waterhouse, A., Bertoni, M., Bienert, S., Studer, G., Tauriello, G., Gumienny, R., ... \& Schwede, T. (2018). SWISS-MODEL: homology modelling of protein structures and complexes. Nucleic Acids Research, 46.

Wiseman, R. L., Zhang, Y., Lee, K. P. K., Harding, H. P., Haynes, C. M., Price, J., ... \& Ron, D. (2010). Flavonol Activation Defines an Unanticipated Ligand-Binding Site in the Kinase-RNase Domain of IRE1. Molecular Cell, 38(2), 291-304.

Yin, Z., Machius, M., Nestler, E. J., \& Rudenko, G. (2017). Activator protein-1: Redox switch controlling structure and DNA-binding. Nucleic Acids Research, 45(19), 11425-11436.

Yoshida, H., Matsui, T., Yamamoto, A., Okada, T., \& Mori, K. (2001). XBP1 mRNA is induced by ATF6 and spliced by IRE1 in response to ER stress to produce a highly active transcription factor. Cell, 107(7), 881-891.

Zhou, J., Liu, C. Y., Back, S. H., Clark, R. L., Peisach, D., Xu, Z., \& Kaufman, R. J. (2006). The crystal structure of human IRE1 luminal domain reveals a conserved dimerization interface required for activation of the unfolded protein response. Proceedings of the National Academy of Sciences of the United States of America, 103(39), 14343-14348. 